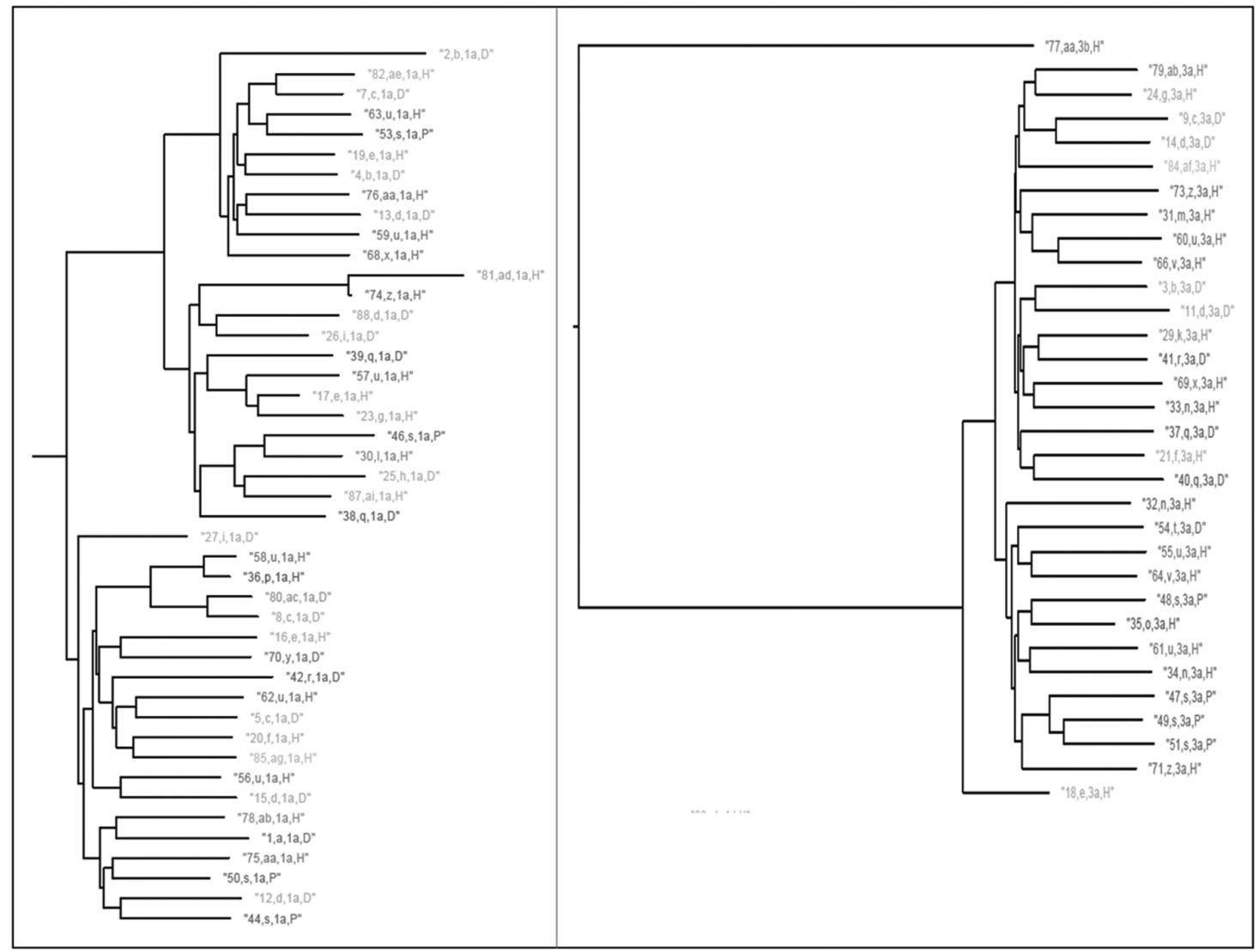

Abstract IDDF2019-ABS-0127 Figure 1 Phylogenetic tree analysis of TB reach samples

used in combination with the BioRobot MDx instrument. Multiple data analyses were performed using phylogenetic approaches.

Results A total of $98 \mathrm{HCV}$ positive samples were found during the study, including 51 from homeless hostels (52.6\%), 30 from drug treatment centres (30.9\%), and 16 from prison $(16.5 \%)$. Only 88 of the 98 samples had sufficient concentration (viral load $>100,000 \mathrm{IU} / \mathrm{ml}$ ) to be further processed through NGS platform in attempt to generate more extensive viral genomes with high read-depth coverage. From the 88 complete HCV genomes assembled, 50/88 (56.8\%) were genotype 1 , followed by $32 / 88$ (36.4\%) genotype 3 , 4/88 (4.5\%) genotype 2 , and $1 / 88(1.1 \%)$ genotype 4 and 6 . Phylogenetics analysis showed a high level of similarity of cases across venues suggests potential continuing ongoing active transmission within these overlapping populations and between venues. There was one cluster of potential within venue transmission found among participants who were recruited in prison. (figure 1)

Conclusions The application of NGS can help to visualise the transmission network among infected individuals, especially between high-risk groups. Increased detail on injecting networks alongside sequencing data could help guide to identify and treat cases and to reduce transmission amongst these networks. If all cases of hepatitis $\mathrm{C}$ in an area were to be routinely sequenced it may be possible to identify larger chains of transmission guiding public health activities to reduce transmission.

\section{IDDF2019-ABS-0128 FUNCTIONAL MECHANISM OF VASCULAR ENDOTHELIAL GROWTH FACTOR IN PROMOTING THE GROWTH OF INTRAHEPATIC CHOLANGIOCARCINOMA}

Yifei Wang ${ }^{*}$, Ruiming Liang, Guangyan Wei, Hongpeng Chu. The First Affiliated Hospital of Sun Yat-sen University, China

\subsection{6/gutjnl-2019-IDDFabstracts.93}

Background To investigate the role of vascular endothelial growth factor (VEGF) and VEGF receptors (VEGFRs) on cell growth of intrahepatic cholangiocarcinoma (ICC).

Methods Western blotting was performed to detect the expression of VEGF in tumour tissues and paired normal tissues from twelve patients with ICC. ICC cell line Huh28 was treated with exogenous recombinant human VEGF (rhVEGF). Cell growth was evaluated by cell counting, proliferation was detected by BrdU cell proliferation assay, and apoptosis was detected by flow cytometry. The expression of VEGF receptors VEGFR1/VEGFR2 in Huh28 cells after rhVEGF treatment was detected by Western blotting. VEGFR1 and VEGFR2 were blocked by specific antibodies, and cell apoptosis was examined by apoptosis-ELISA assay. The stably knockdown VEGFR2 cell line Huh28-shVEGFR2 (experimental group) and the control cell line Huh28-shNC (control group) were established using shRNA lentivirus. Subcutaneous tumour models in ten nude mice with Huh28-shVEGFR2 and Huh28shNC were used to observe tumour growth. 
A

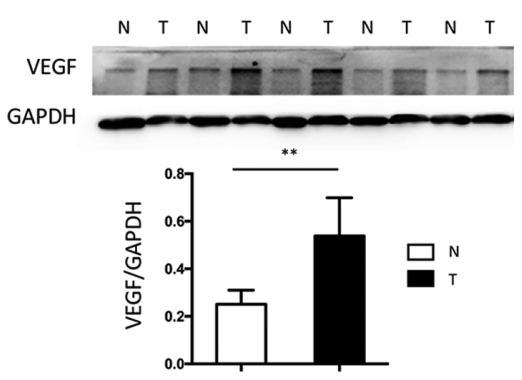

D

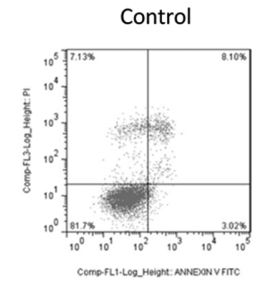

E

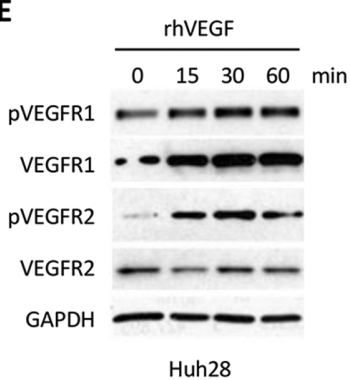

SM

Huh28

$\mathbf{F}$
B

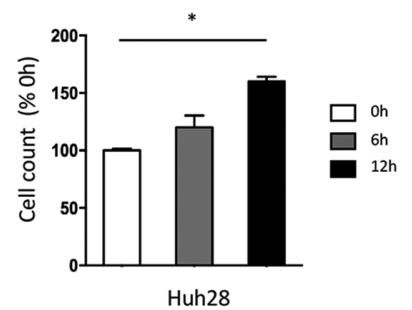

C
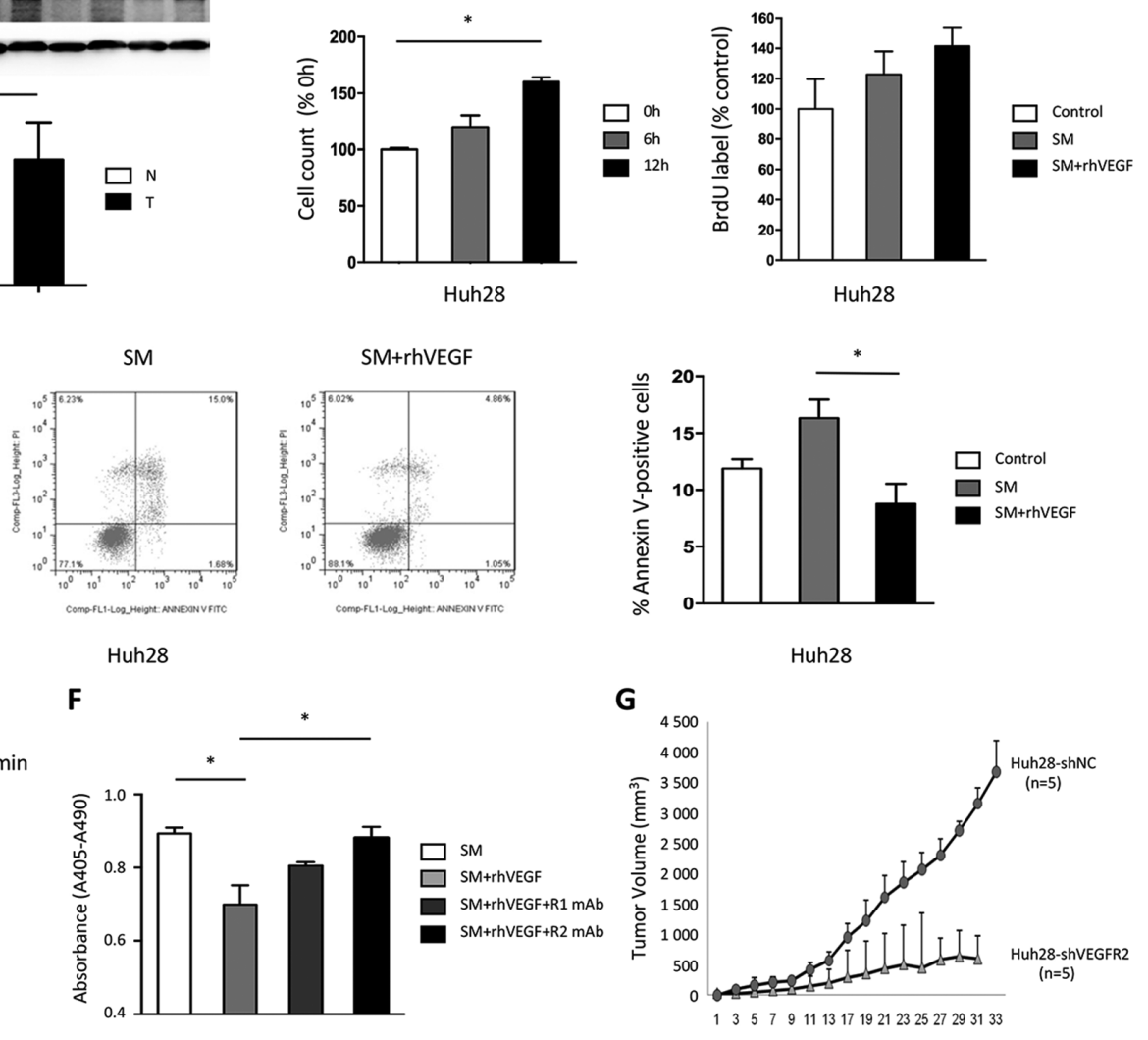

Huh28

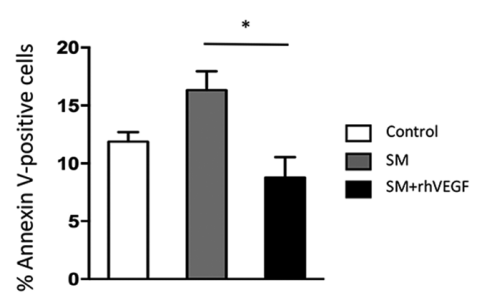

Huh28

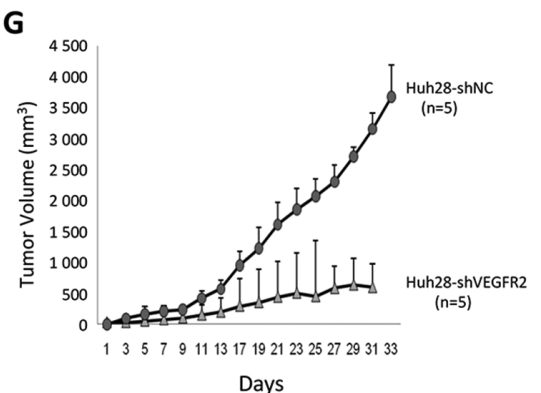

\section{Abstract IDDF2019-ABS-0128 Figure 1}

Results The protein expression of VEGF was up-regulated in ICC tumour tissues than in matched normal tissues $(P<0.01)$ (figure $1 \mathrm{~A})$. Exogenous rhVEFG could promote the growth of Huh28 cells, suppressed cell apoptosis, without significant effect on cell proliferation ability (figure 1 B-D). The expression of phosphorylated VEGFR1 and VEGFR2 in Huh28 cells were up-regulated after rhVEGF treatment $(P<0.05)$ (figure $1 \mathrm{E}$ ). VEGFR2 antibody could significantly reverse the anti-apoptosis effect of rhVEGF $(P<0.05)$, while VEGFR1 antibody did not (figure $1 \mathrm{~F}$ ). Subcutaneous tumour models indicated that tumour growth in the experimental group (Huh28-shVEGFR2) was significantly inhibited compared with the control group (Huh28shNC) (figure $1 \mathrm{G}$ ).

Conclusions VEGF promotes ICC cells growth through inhibiting apoptosis of ICC cells in a VEGFR2-dependent signalling pathway.

\section{IDDF2019-ABS-0140 ATTENUATION OF DIETHYLNITROSAMINE INDUCED HEPATIC CANCER IN WISTAR ALBINO RATS BY MADHUCA LONGIFOLIA EXTRACT VIA KNOCKDOWN OXIDATIVE STRESS AND INFLAMMATION}

Manvendra Singh*. HMFA MIET, Abdul Kalam Technical University, Handia, India
Background Hepatocellular cancer occurred due to hepatocytes damage and inflammation. Interleukin as a pleiotropic cytokine in the immune system plays a pivotal role in the onset and progression of tumours. Emphasizing on approach to downregulate the cytokines might be an effective boon for the treatment of hepatocellular carcinoma. Madhuca longifolia used as a traditional plant in the treatment of hepatocellular carcinoma. So focused on this scrutiny, we evaluate the therapeutic potential of the aqueous extract of Madhuca longifolia in Diethylnitrosamine induced hepatic cancer in Wistar albino rats.

Methods To determine the effect of aqueous extract of Madbuca longifolia leaf following Diethylnitrosamine exposure, the antioxidant activity was measured by the enzymatic and nonenzymatic antioxidants. In addition Proinflammatory cytokines and inflammatory mediators, namely TNF-a, IL-1b, IL-10 and $\mathrm{NF}-\mathrm{kb}$ were also assessed.

Results The finding of the current study reveals that DEN treatment significantly downregulated the content of antioxidant parameter (SOD, catalase, GPx, GSH, G6PD and vitamin C) and upregulated the level of proinflammatory cytokines. Administration of aqueous extract of Madhuca longifolia attenuated the antioxidant parameter, cytokines and inflammatory mediators in rats.

Conclusions These study clearly highlight the chemopreventive prospective of Madhuca longifolia aqueous extract against DEN-induced Hepatic cancer by knockdown oxidative stress and inflammation. 